\title{
El derecho a la Consulta previa a los pueblos indígenas en el Sistema Interamericano de Derechos Humanos. Casos de estudio: Ecuador y Colombia ${ }^{1}$
}

\author{
The Right of Indigenous Peoples to Prior Consultation in the Inter-American Human \\ Rights System. Case studies: Ecuador and Colombia
}

Recibido: 27 de febrero de 2018 - Revisado: 2 de noviembre de 2018 - Aceptado: 15 de diciembre de 2018

Sorily Carolina Figuera Vargas ${ }^{2}$

Meylin Heleana Ortiz Torres ${ }^{3}$

\section{Resumen}

El presente estudio tiene como principal objetivo analizar los aportes del Convenio No. 169 sobre Pueblos Indígenas y Tribales en Países Independientes de la Organización Internacional del Trabajo (OIT), acerca del derecho a la consulta previa como herramienta de protección de los pueblos indígenas en el Sistema Interamericano de Derechos Humanos. Este análisis se fundamentó en la metodología cualitativa, se atendió al enfoque analítico-descriptivo, se utilizaron herramientas como el análisis jurisprudencial, y se enfocó en la sentencia de la Corte Interamericana Sarayaku vs. Ecuador. Finalmente, se dedujeron algunas realidades jurídicas relativas a las consecuencias de acoger a la consulta previa en los ordenamientos jurídicos de Ecuador y Colombia; así como, su incidencia en la realidad socio - económico de estos Estados y en su población indígena.

\section{Palabras clave}

Pueblos indígenas, consulta previa, Convenio No. 169 de la OIT, Corte Interamericana.

\begin{abstract}
This article analyzed the contributions of ILO Convention 169 concerning Indigenous and Tribal Peoples in Independent Countries, regarding the right to prior consultation as a tool for the protection of indigenous peoples in the Inter-American Human Rights System. This analysis was based on a qualitative methodology, following the analyticaldescriptive approach and using tools such as jurisprudential analysis. The research focused, particularly, on studying the sentence of the Inter-American Court of Human Rights in the case of Sarayaku v. Ecuador. From this sentence, some legal realities can be deduced that foresee the consequences of adopting the prior consultation in the legal systems of Ecuador and Colombia, as well as its impact on the socio-economic reality of those states and their indigenous peoples.
\end{abstract}

Keywords

Indigenous people, prior consultation, ILO Convention 169, Inter-American Court of Human Rights.

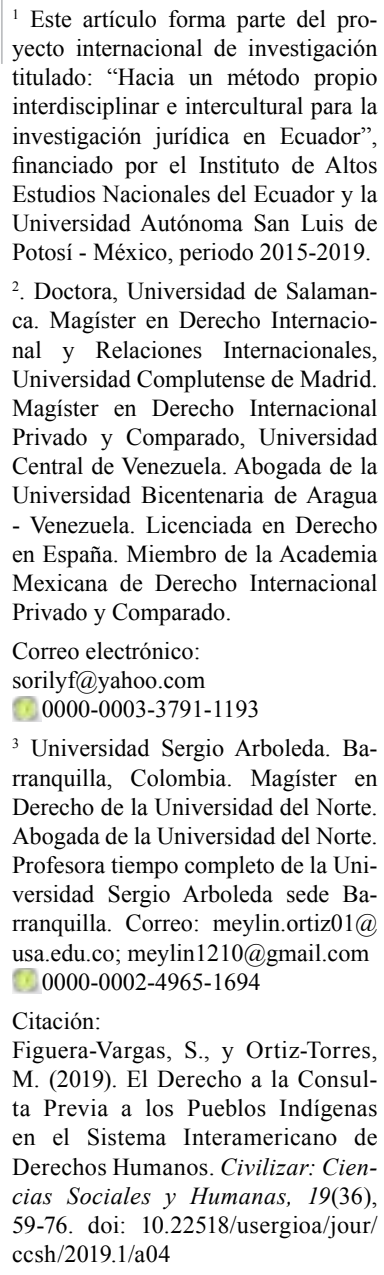

${ }^{1}$ Este artículo forma parte del proyecto internacional de investigación titulado: "Hacia un método propio interdisciplinar e intercultural para la investigación jurídica en Ecuador", financiado por el Instituto de Altos Estudios Nacionales del Ecuador y la Universidad Autónoma San Luis de Potosí - México, periodo 2015-2019.

2. Doctora, Universidad de Salamanca. Magíster en Derecho Internacional y Relaciones Internacionales, Universidad Complutense de Madrid. Magíster en Derecho Internaciona Privado y Comparado, Universidad Central de Venezuela. Abogada de la Universidad Bicentenaria de Aragua - Venezuela. Licenciada en Derecho en España. Miembro de la Academia Mexicana de Derecho Internacional Privado y Comparado.

Correo electrónico: sorilyf@yahoo.com 0000-0003-3791-1193

${ }^{3}$ Universidad Sergio Arboleda. Barranquilla, Colombia. Magíster en Derecho de la Universidad del Norte. Abogada de la Universidad del Norte. Profesora tiempo completo de la Universidad Sergio Arboleda sede $\mathrm{Ba}-$ rranquilla. Correo: meylin.ortiz01@ usa.edu.co; meylin1210@gmail.com 0000-0002-4965-1694

Citación:

Figuera-Vargas, S., y Ortiz-Torres, M. (2019). El Derecho a la Consulta Previa a los Pueblos Indígenas en el Sistema Interamericano de Derechos Humanos. Civilizar: Ciencias Sociales y Humanas, 19(36), 59-76. doi: 10.22518/usergioa/jour/ $\operatorname{ccsh} / 2019.1 / \mathrm{a} 04$ 


\section{Introducción}

Este artículo analiza la consulta previa, de acuerdo con los lineamientos del Convenio No. 169 de la OIT. El interés del presente estudio surge de la necesidad de analizar los elementos y aportes de dicho convenio en la realidad jurídica de los pueblos indígenas del contexto interamericano.

Desde el punto de vista del estudio del derecho internacional de derechos humanos, en especial desde el trabajo adelantado por el Sistema Interamericano de Derechos Humanos a través de la Corte Interamericana, resulta relevante analizar el deber de consulta que tienen los Estados con los pueblos indígenas que aún se encuentran en constante lucha por sus derechos.

Las principales problemáticas son la revisión a profundidad de los elementos jurídicos que brinda el Derecho Internacional y ahondar en las falencias estructurales al interior de los ordenamientos jurídicos de los Estados de Latinoamérica, que impiden el desarrollo de las garantías recibidas por los pueblos indígenas en el plano internacional.

La consulta previa a pueblos indígenas ${ }^{1}$ y otros grupos étnicos es un tema objeto de controversia al interior de la academia, en el marco de los planteamientos del Derecho Internacional de los Derechos Humanos(Morris, Rodríguez, Orduz y Buriticá, 2009). El estudio de la consulta en el Derecho Internacional es más visible a partir de las obligaciones que los Estados adquirieron con la adopción del Convenio No. 169 de la OIT $^{2}$; este surge como instrumento que regula lo relacionado con los intereses sociales y económicos frente a la supervivencia de los pueblos indígenas y demás grupos étnicos (Morris et al., 2009, p. 5).

Cabe destacar que el desarrollo de los derechos de los pueblos indígenas en el marco del sistema de derechos humanos, surge al interior de la Organización Internacional del Trabajo (OIT). Esto obedece a la relación directa entre la OIT y los trabajadores indígenas, ya que la lucha por sus derechos inició con la reivindicación de su condición de trabajadores al interior de las colonias europeas (Rodríguez Van der Hammen, 2013). Es pertinente establecer que el análisis de dicho instrumento internacional se comprende bajo la relación histórica entre la OIT y los países latinoamericanos desde tiempos coloniales.

Por lo anterior, el texto pretende dar respuesta a los siguientes interrogantes: (1) ¿En qué radica la importancia del Convenio 169 de la OIT en el marco de la jurisprudencia del Sistema Interamericano de Derechos Humanos? (2) ¿Cuál es la relevancia del caso Sarayaku vs. Ecuador, acerca del principio de consulta desde el derecho internacional de los derechos humanos? y (3) ¿En qué consiste la recepción del Convenio 169 de la OIT en cuanto al ejercicio del deber de consulta adelantado en países como Colombia y Ecuador, al interior de sus ordenamientos jurídicos, frente a los intereses y derechos de los pueblos indígenas?

El presente análisis se basó en el método cualitativo, atendió al enfoque analítico-descriptivo, utilizó herramientas como el análisis jurisprudencial, y tuvo en cuenta los aportes del derecho comparado para la descripción de los casos Ecuador y Colombia.

\section{EI Convenio No. 169 de la OIT, su relevancia en el estudio de la consulta previa a los pueblos indígenas}

El Convenio No. 169 de la Organización Internacional de Trabajo sobre Pueblos Indígenas y Tribales del año 1989 representó un viraje significativo de la concepción global que los Estados tenían acerca de las etnias indígenas. Para señalar la ideología que sustenta este Convenio, es necesario acudir a su preámbulo, que desarrolla las aspiraciones de los pueblos indígenas a: (a) asumir el control 
de sus propias instituciones y formas de vida; (b) asumir su desarrollo económico; y (c) mantener y fortalecer sus identidades, lenguas y religiones, dentro del marco de los Estados que forman parte (Anaya, 2005). Se puede afirmar que este instrumento internacional tiene como premisa universal comprometer a los Estados a respetar todas las aspiraciones de sus pueblos indígenas en relación con las decisiones que los involucran, esto en concordancia con su preámbulo (Figuera, 2015).

Entre los aportes significativos del Convenio No. 169 de la OIT se encuentra el mecanismo de la consulta previa $^{3}$, que se impulsa con la pretensión de acercar a los gobiernos, las organizaciones indígenas y las organizaciones no gubernamentales. La consulta nace como un mecanismo-derecho de las comunidades étnicas y una obligación en cabeza del Estado que debe ejercerse frente a las leyes, los actos administrativos o aquellos proyectos de desarrollo que de alguna manera pueden llegar afectar a los pueblos indígenas y tribales (Rodríguez Van der Hammen, 2013, p. 8).

Es pertinente destacar que el deber de consulta que expone el Convenio No. 169 no se relaciona solo con proyectos de desarrollo en particular, sino que se refiere a temas que se discuten en el ejercicio de gobernanza; la finalidad es impulsar la participación activa de los pueblos indígenas y tribales en la vida pública (Organización Internacional del Trabajo, 2014).

No obstante, el Convenio presenta la obligación de consulta que tienen los gobiernos hacia los pueblos indígenas, con relación al aprovechamiento de los recursos naturales situados en sus tierras ancestrales. En este orden de ideas, se consolida el compromiso por el reconocimiento del derecho de participación de los pueblos interesados en las decisiones de los Estados cuando se trate de la utilización, administración y conservación de los recursos naturales, los cuales además deberán ser protegidos de forma especial (Rodríguez Van der Hammen, 2013, p. 10).

\section{La consulta previa a pueblos indígenas. Un análisis de la jurisprudencia de la Corte Interamericana de Derechos Humanos y su sustento en el Convenio 169 de la OIT}

En el estándar internacional del derecho a la consulta previa, resulta importante destacar los aportes verificados dentro del Sistema Interamericano de Derechos Humanos, donde los derechos de los pueblos indígenas se han venido interpretando y protegiendo mediante instrumentos como la Convención Americana de Derechos Humanos y la Declaración Americana de Derechos y Deberes del Hombre ${ }^{4}$.

La Declaración Americana sobre los Derechos de los pueblos indígenas (OEA, 2015) resume la necesidad de cristalizar en lo jurídico la importancia del consentimiento informado y la participación de los pueblos indígenas en los proyectos de desarrollo que involucran sus tierras y recursos ${ }^{5}$.

A nivel regional, la dinámica de protección de los derechos de los pueblos indígenas con relación a temáticas como el derecho a la consulta previa, se lleva a cabo conforme a los lineamientos de la función cuasi judicial ${ }^{6}$ de la Comisión Interamericana de Derechos Humanos o la función jurisdiccional de la Corte Interamericana de Derechos Humanos.

En este sentido, la mayor contribución se encuentra en el trabajo de la Corte Interamericana de Derechos Humanos en su interpretación y protección de los derechos de los pueblos indígenas. En dichas sentencias destaca la consulta previa como mecanismo de protección del derecho a la diversidad étnica y cultural (Rodríguez Van der Hammen, 2013).

Es posible evidenciar los aportes de la Corte Interamericana de Derechos Humanos a través de una línea jurisprudencial, que ha abordado temas en torno al deber de consulta que tienen los Estados hacia sus pueblos indígenas. 
Así pues, en una primera sentencia se pronunció sobre el Caso Mayagna Awas Tigni vs. Nicaragua, haciendo referencia a la responsabilidad internacional del Estado por la falta de delimitación del territorio de la comunidad Mayagna Awas Tigni. Al respecto, la Corte manifestó que:

Entre los indígenas existe una tradición comunitaria sobre una forma comunal de la propiedad colectiva de la tierra, en el sentido de que la pertenencia de ésta no se centra en un individuo sino en el grupo y su comunidad. Los indígenas por el hecho de su propia existencia tienen derecho a vivir libremente en sus propios territorios; la estrecha relación que los indígenas mantienen con la tierra debe de ser reconocida y comprendida como la base fundamental de sus culturas, su vida espiritual, su integridad y su supervivencia económica. Para las comunidades indígenas la relación con la tierra no es meramente una cuestión de posesión y producción sino un elemento material y espiritual del que deben gozar plenamente, inclusive para preservar su legado cultural y transmitirlo a las generaciones futuras. (Consejo de Estado, Sentencia 00873, 2015).

La Sentencia Mayagna Awas Tigni vs. Nicaragua representó un avance de la Corte por el reconocimiento de los derechos ancestrales de los pueblos indígenas y la exposición del criterio de la ocupación histórica como título original (Ariza, 2013). Seguido, en el Caso de la Comunidad Moiwana vs. Suriname, la Corte expuso que:

La relación de la comunidad N'djuka con su tierra tradicional es de vital importancia espiritual, cultural y material. Para que la cultura mantenga su integridad e identidad, los miembros de la comunidad deben tener acceso a su tierra de origen. Los derechos a la tierra en la sociedad N'djuka existen en varios niveles, y van desde los derechos de la comunidad entera hasta los del individuo. Los derechos territoriales más amplios están depositados en todo el pueblo, según la costumbre N'djuka; los miembros de la comunidad consideran que dichos derechos son perpetuos e inalienables (Párrafo 86.6).
Se observa que la Corte usó el mismo argumento expuesto en el caso Mayagna Awas Tigni, en lo relativo a la estrecha relación que mantienen los pueblos indígenas con la Tierra, basada en su cultura. Del mismo modo, lo hizo en el caso Saramaka vs. Suriname, ${ }^{7}$ en el cual estableció la necesidad de ir más allá de la consulta, al evidenciar la necesidad de obtener el consentimiento libre, previo e informado (Morris et al, 2009). Rodríguez (2014) considera que "el antecedente más importante del consentimiento libre, previo e informado es precisamente el caso de Saramaka vs. Suriname" (p. 206), en el cual la Corte determinó que el consentimiento no sólo tiene como finalidad el proceso de consulta, sino que en los casos relacionados con proyectos de gran escala, su relevancia radica en el valor para la subsistencia del grupo y la protección de las minorías.

Así también, en la Sentencia sobre el Caso Yakye Axa vs. Paraguay, la Corte se extendió al tema de reparaciones más allá de las fuentes del Derecho internacional, ordenando la devolución de tierras y otras formas de reparaciones no pecuniarias, así como indemnizaciones (Morris et al, 2009). Entre otros aportes, estableció una ampliación del derecho a la propiedad colectiva exponiendo que: "están vinculados estrechamente a la garantía de este derecho: el derecho a la vida, el derecho a la identidad étnica, el derecho a la cultura y a la recreación de la misma, el derecho a la integridad y supervivencia como comunidad indigena" (Corte IDH, núm. h, párrafo 121).

En el Caso de la Comunidad Garifuna de Punta Piedra y Sus Miembros vs. Honduras, la Corte insistió en el carácter previo del proceso de consulta y constató que el Estado no llevó a cabo un proceso adecuado y efectivo que garantizara el derecho a la consulta de la Comunidad Garífuna de Punta Piedra, frente al proyecto de exploración en su territorio (Corte IDH, párrafo 224). Asimismo, la relevancia del caso consistió en la falta del deber del Estado de armonizar la legislación y reglamentación interna respecto de las etapas previas de la consulta. 
En este mismo orden, en el Caso Pueblos Kaliña y Lokono vs. Surinam, la Corte encontró que, si bien la concesión minera se dio en el año 1958, las actividades de extracción se iniciaron casi cuarente años después; para la fecha ya se precisaba el lugar donde se realizarían las actividades extractivas. Así las cosas, la garantía de participación debió hacerse al inicio de la extracción o explotación minera, lo que no ocurrió en el presente caso.

La Corte estimó que, pese a la normativa interna que, reconocía los intereses de los derechos de los pueblos indígenas y tribales al otorgar concesiones forestales y mineras, esto no incluía el derecho a la participación efectiva. En adición, el Estado indicó que se estaba elaborando un "protocolo de consulta previa, libre e informada" que, sin embargo, no fue adoptado hasta la fecha.

Hasta este punto, se puede decir que, a través de su jurisprudencia, la Corte Interamericana desarrolló una construcción teórica del carácter y los componentes acerca del deber de la consulta previa, a saber, la relación de los pueblos indígenas con la Tierra, su derecho de propiedad comunal y los derechos territoriales que se derivan de estos. Sin embargo, con relación a una descripción amplia de la consulta previa y su incidencia en los ordenamientos constitucionales, se hizo efectiva solo hasta el caso Sarayaku vs Ecuador, en el que la Corte estableció de manera directa y concreta la relevancia de esta figura, que consagra el derecho de participación de los pueblos indígenas.

En el Caso Sarayaku vs. Ecuador, la Corte Interamericana de Derechos Humanos estableció cómo los diversos Estados miembros de la Organización de Estados Americanos (OEA) han incorporado el estándar internacional en lo relativo al deber de consulta previa a través de su normatividad interna y por medio de sus más altos tribunales de justicia, , principalmente ajustado al planteamiento de la situación jurídica de cada país frente al cumplimiento del Convenio 169 de la OIT.
Thomas M. Antkowiak señala la importancia de la Sentencia Sarayaku, al expresar la relevancia del Convenio 169 de la OIT y la inserción de la consulta como principio general del derecho internacional, así:

Sarayaku dedicó una gran atención a la línea de base de la participación efectiva: la obligación del Estado de consultar a los pueblos indígenas antes de que comiencen los proyectos. Saramaka ya había afirmado que esto constituía un "derecho a la consulta" para las comunidades cuyas tierras tradicionales fueron amenazadas. La Corte en Sarayaku destacó que la ley ecuatoriana "reconoció plenamente este derecho". Sarayaku luego inspeccionó la ley regional sobre este tema, y reconoció su estatus en los instrumentos internacionales, tales como el Convenio $\mathrm{N}^{\circ} 169$ de la OIT. La apreciación de la Corte trató de establecer el derecho a la consulta, no sólo como una norma protegida en la Convención Americana, sino también como un "principio general del Derecho internacional. (Antkowiak, 2014, p. 157).

El caso Sarayaku dejó ver cómo, mediante su jurisprudencia, la Corte Interamericana desmitificó el significado de la consulta previa y amplió de forma explícita los derechos acerca de las medidas legislativas y administrativas que afecten a los pueblos indígenas. En esta sentencia, la Corte otorgó carácter de garantía fundamental a la consulta previa, para garantizar el alcance del derecho a la participación de los pueblos indígenas en las decisiones que afectan sus derechos. Así, se remitió expresamente al reconocimiento del derecho a la consulta previsto en el Convenio 169 de la OIT. Además, insistió en que las consultas debían hacerse en el marco de un buen flujo de comunicación entre los actores interesados, mediante procesos ajustados a la cultura de la población objeto de la indagación, con el fin de lograr un acuerdo. Así, el caso de Sarayaku centró su análisis en los pilares relativos: (a) al carácter previo de la consulta, (b) la buena fe y finalidad de llegar a un acuerdo, (c) la consulta adecuada y accesible, (d) Estudio del impacto ambiental, y (e) consulta informada. 
Sobre el carácter previo, expresó que se debía consultar siguiendo las costumbres del pueblo indígena y que esto se debía hacer en las primeras etapas del plan de desarrollo o de inversión. En lo relacionado con la buena fe y la finalidad de lograr un acuerdo, insistió que la consulta no era un trámite formal, sino que su naturaleza obedecía a un instrumento de participación. Así pues, la dinámica de la consulta debía regirse bajo principios de confianza y respeto de los actores involucrados. Aclaró además, que la buena fe exigía que no hubiese ningún elemento de coerción por parte de los mismos, que era inaceptable el ánimo de desintegración en las comunidades que en ocasiones se ejercía sobre sus líderes indígenas (Corte IDH, párr. 186).

Asimismo, determinó que para obtener una consulta adecuada y accesible se requería de un procedimiento culturalmente adecuado, conforme a las tradiciones del pueblo indígena. Haciendo alusión a la Comisión de Expertos en Aplicación de Convenios y Recomendaciones de la OIT, la Corte recalcó que un procedimiento apropiado debía ser coherente con la finalidad de la consulta, pues no existía un único modelo. Para ello se debería tener en cuenta las circunstancias nacionales y la de los pueblos indígenas, como las medidas consultadas; a su vez, especificó que la consulta tenía una dimensión temporal que debía ser análoga con la medida propuesta.

Luego, la Corte estableció que el estudio de impacto ambiental conforme al artículo 7.3 del Convenio 169 de la OIT señalaba el deber de los gobiernos por proporcionar los estudios de la mano de los pueblos indígenas, para valorar la incidencia social, espiritual, cultural y ambiental que puedan llegar a presentar la ejecución. A su vez, la finalidad del estudio consistía en determinar el daño o impacto que pudiese tener el proyecto de desarrollo e inversión sobre el territorio y la comunidad (Hillón, 2014). En relación con el nivel de información de la Consulta, la Corte expresó que el pueblo indígena debía tener conocimiento de los riesgos que presentaba el plan de desarrollo o de inversión, incluyéndose riesgos de tipo ambiental y lo correspondiente a salubridad.

Autores como Bogdandy, Morales Antoniazzi y Figuera Vargas, han sostenido que en la Sentencia Sarayaku, la Corte hizo un verdadero ejercicio de Ius Constitucionale Comune, al citar doce sentencias de las Cortes Constitucionales de Estados que conforman el sistema interamericano, en las que se pronunciaban a favor del derecho a la consulta previa que tenían sus pueblos indígenas (Figuera, 2015). Lo anterior permite evidenciar una vez más el enlace entre el Ius Constitucionale Comune y la internacionalización del derecho constitucional, que busca superar la realidad regional, para ahondar en otros aspectos de común interés en el mundo del Sur Global (Bogdandy, Salazar, Morales y Ebert, 2018, p. 9).

\section{La consulta previa a los pueblos indígenas: un estudio comparado entre Ecuador y Colombia}

El caso Sarayaku es un estudio de la consulta previa a pueblos indígenas, desarrollado por la Corte Interamericana de Derechos Humanos, que hace un recorrido legislativo y jurisprudencial del estándar internacional de la aplicación del Convenio No. 169 de la OIT en los Estados firmantes ${ }^{8}$ y no firmantes ${ }^{9}$. Resulta pertinente rescatar los casos de Colombia y Ecuador, en cuanto al ejercicio del deber de consulta adelantado por estos Estados, así mismo, las controversias suscitadas al interior de sus ordenamientos jurídicos, frente a los intereses y derechos de los pueblos indígenas.

\section{Ecuador}

En Ecuador, la Constitución de $1998^{10}$ consideró la consulta previa como un requisito para la intervención en el territorio de los pueblos indígenas (Saavedra, 2011). Antes de ello se verificaba una problemática en la que el resultado de la consulta no influía en la realiza- 
ción de dicha intervención, como consecuencia, el Estado y las empresas hicieron toda clase de acciones para simular el agotamiento del requisito; entre ellas, conformar asambleas con los pueblos indígenas para mostrar el proyecto sin importar si lo comprendían o si, luego, eran aceptados o no. En vista de lo anterior surgió la necesidad de contemplar en el ordenamiento jurídico del Ecuador, la figura del consentimiento previo e informado; no obstante, el consentimiento no se incorporó en la Constitución de $2008^{11}$.

Sobre el desarrollo de la protección constitucional a los pueblos indígenas del Ecuador, se dice que la Constitución de 2008 hace dieciséis referencias al carácter intercultural del Estado, mientras que el plurinacional solo se menciona tres veces. En palabras de Fernando García (2014) "estos datos evidencian un desigual desarrollo de ambos temas" (p. 77). Además, cabe resaltar que la concepción de tierra y territorio de los pueblos indígenas del Ecuador, contradice de tajo la concepción del Estado, pues es para ellos la pachamama ${ }^{12}$ y origen de la vida. En cambio, el Estado ve a la tierra con fines de explotación y acumulación. García (2014) insiste que esta realidad jurídica favorece a los poderes económicos y políticos $\mathrm{y}$, desafortunadamente, entre ellos no figuran los pueblos indígenas.

Pese a lo antes expuesto, cabe destacar que el reconocimiento constitucional del derecho a la consulta es un avance en los derechos de los pueblos indígenas; sin embargo, el verdadero retroceso, se presentó cuando el gobierno logró la aprobación de los decretos 3401 del año $2002^{13}$ y el Decreto 1040 del $2008^{14}$. La realidad ecuatoriana ofrece un panorama desalentador en cuanto a la aplicación de la consulta previa frente a los proyectos de inversión, pues no ha sido reconsiderada desde la institucionalidad estatal, ni en el presupuesto público (DPLF y Ofxam, 2011).
Una de las causas principales es la falta de participación (o consulta) que han tenido los pueblos indígenas del Ecuador en la adopción de normas socio-económicas de gran trascendencia para el país (Saavedra, 2011). La participación política del Ecuador, se manifiesta a través de la Confederación de Nacionalidades Indígenas del Ecuador (CONAIE), que en 1986 se conformó de comunidades, centros, federaciones y confederaciones de todos los pueblos indígenas del país (Saavedra, 2011).

Uno de los ejemplos que mejor ilustran la causa es la falta de consulta previa a los pueblos indígenas durante el proceso legislativo que incorporó los requisitos de la consulta previa en la Ley de Minería, aprobada el 29 de enero de 2009 (Saavedra, 2011).

A propósito de lo mencionado, en la Sentencia sobre el caso Sarayaku vs. Ecuador, la Corte Interamericana, hizo un recuento en el marco de la aprobación del Convenio 169 de la OIT, en el cual citó varios casos emblemáticos para la situación del Ecuador, dentro de los cuales cabe destacar la Sentencia No. 001-10-SINCC que corresponde a la demanda de inconstitucionalidad de la Ley de Minería.

$\mathrm{Al}$ respecto, se ha considerado que la Ley de Minería desnaturaliza el derecho de participación, entendiéndolo como un proceso de información sobre los impactos de la actividad minera, la obligación del concesionario ante la sociedad y autoridades, desde que se otorga la concesión minera y en el transcurso del proceso (Carrión, 2012). Sobre la promulgación de dicha ley, las reacciones de las organizaciones indígenas del Ecuador no se hicieron esperar e interpusieron demanda de constitucionalidad con relación a la violación del derecho de consulta previa frente al Convenio 169 de la OIT, la Declaración de Derechos de los Pueblos Indígenas y la Constitución ecuatoriana; ya que esta ley no fue consultada a los pueblos indígenas, en razón de la afectación que recibirían en sus 
territorios donde se realizarían actividades de extracción minera.

Por su parte, el ejecutivo se defendió exponiendo que hizo un proceso de consulta de acuerdo con lo establecido en el artículo 389 de la Constitución. Sin embargo, esta norma constitucional se refiere a la consulta ambiental. Así las cosas, la Corte Constitucional del Ecuador estableció la diferencia entre la consulta pre legislativa $^{15}$ y la consulta ambiental, indicando que la consulta pre legislativa no es un mero procedimiento formal, sino un derecho constitucional de carácter colectivo que es parte de los elementos indispensables para la aprobación de las leyes (Carrión, 2012, p. 52). Sobre este caso vale la pena traer la reflexión de Patiño (2014), quien al citar el reglamento de consulta y participación para las actividades en tierras comunitarias del Ecuador, expresa el punto de vista del Estado, la empresa y los pueblos indígenas acerca del deber de consulta:

El derecho-deber de consulta genera conflictos desde su concepción, ya que, por una parte, los pueblos indígenas, consideran la consulta previa como aquella facultad que tienen para tomar decisiones sobre todos los asuntos que les afecten, incluidas las actividades extractivas en sus tierras y territorios; en cambio, visto desde los órganos del poder estatal y la empresa privada, generalmente hay una coincidencia respecto a que la consulta previa es una herramienta para el establecimiento de medidas de prevención y remediación de impactos como los socio-ambientales manifestado que la potestad de decidir es exclusiva del Estado. (pp. 71-72).

No obstante, en Ecuador se evidenció un avance legislativo en relación con este tema. El Código Orgánico del Ambiente, promulgado en abril de 2017, determina en el numeral 7 del artículo 8, que es una responsabilidad ambiental del Estado, sin perjuicio de otras establecidas por la Constitución y la ley, garantizar que las decisiones o autorizaciones estatales que puedan afectar al ambiente sean consultadas con la comunidad. A esta se debe brindar información amplia y de forma oportuna, según lo previsto en el orden normativo de ese país. Previsión que se reitera en el mismo cuerpo legal, en el numeral 6 del artículo 9, dentro del principio de acceso a la información, participación y justicia en materia ambiental.

\section{Colombia}

En el caso de Colombia, cabe recurrir al análisis de la jurisprudencia de la Corte Interamericana de Derechos Humanos. Colombia se destaca en el contexto interamericano por un amplio desarrollo de derechos y garantías a los pueblos indígenas, pese a que es un país donde estos pueblos representan en términos poblacionales, un grupo minoritario, pues según datos estadísticos en el territorio Colombiano habitan alrededor de 45 millones de personas, de las cuales solo el 3,4\% está representado por la comunidad indígena ${ }^{16}$. Ellos se han enfrentado a las diferentes problemáticas que acarrea su reconocimiento y posterior reivindicación de sus derechos como minorías.

Desde la década de los 90, el Estado colombiano ha aceptado acogerse a diferentes instrumentos internacionales que permiten la reivindicación de los derechos de los pueblos indígenas. En la actualidad, las organizaciones y los líderes indígenas continúan su lucha por expresar su acción "dentro" del Estado; el cual "con la Constitución Política de 1991 se instituyó como veedor del carácter multiétnico y pluricultural de la nación colombiana" (Laurent, 2005, pp. 138-159).

En Colombia el desarrollo de los derechos de los pueblos indígenas se da a partir de la Constitución Política de 1991, donde fueron reconocidos como "minoría multicultural" (Corte Constitucional Colombia, Sentencia C-139/196, 1996), para lo que se desplegaron conceptos del Derecho internacional (OIT, Convenio No. 169 sobre Pueblos Indígenas y Tribales en Países Independientes), pues este 
reconocimiento correspondió a la apertura del sistema jurídico colombiano hacia la recepción de garantías y ampliación de derechos para este tipo de comunidades.

Dentro de los referentes legislativos más importantes que resaltar se encuentra la Ley 21 de 1990, mediante la cual se aprobó el Convenio No. 169 sobre pueblos indígenas y Tribales en Países Independientes de la OIT. El compromiso que se adquirió con esta Convención por parte de Colombia envuelve todas las actuaciones que permiten la protección de la identidad cultural de esta comunidad, sus bienes, su trabajo y medio ambiente.

Entre la lista de derechos y garantías que recibió el orden interno colombiano con la aprobación del Convenio 169 de la OIT se encuentra el mencionado derecho a la consulta previa, que nace como un mecanismo de protección de los derechos colectivos indígenas. Así las cosas, se introdujo a los indígenas dentro de la participación política del país ${ }^{17}$ (Constitución política de Colombia, 1991, arts. 171 y 286); a su vez, se reconoció y fomentó el multiculturalismo dentro de los diferentes grupos étnicos e indígenas ${ }^{18}$ (Constitución política de Colombia, 1991, arts. 246, 321, 329 y 330 ).

Dentro de los temas que preocupan a las organizaciones y los organismos en el orden interno de Colombia, se encuentra lo relativo a la consulta de los pueblos indígenas en el contexto de globalización y los enfrentamientos que puedan existir entre el ánimo de progreso económico y la existencia misma de estos grupos sociales.

En este orden de ideas, en Colombia, la Corte Constitucional ha sido quien ha determinado el alcance y dinámica del derecho de consulta, así como también el deber de sortear las distintas problemáticas presentadas en el territorio colombiano con relación a la explotación de recursos naturales y la protección de la identidad cultural de los indígenas, profundizando en algunas aristas que componen el enfrentamiento entre los actores que, al final, participan en la consulta, a saber, instituciones del Estado, pueblos indígenas y empresas.

Rodríguez y Orduz (2012) hicieron un recuento de las pautas jurídicas generales que rigen la consulta según la Corte Constitucional, dejan ver que esto se lleva a cabo desde un enfoque general y que es necesario analizar los desafíos de la consulta en la práctica, y relacionándola con experiencias concretas. Los autores citan la primera aplicación que de la Corte Constitucional colombiana en 2009 bajo la guía de la Corte Interamericana de Derechos Humanos (Corte IDH) acerca del consentimiento libre, previo e informado. Allí afirman que:

[cuando] se trate de planes de desarrollo o de inversión a gran escala, que tengan mayor impacto dentro del territorio de afrodescendientes e indígenas, es deber del Estado no sólo consultar a dichas comunidades, sino también obtener su consentimiento libre, informado y previo, según sus costumbres y tradiciones, dado que esas poblaciones, al ejecutarse planes e inversiones de exploración y explotación en su hábitat, pueden llegar a atravesar cambios sociales y económicos profundos, como la pérdida de sus tierras tradicionales, el desalojo, la migración, el agotamiento de recursos necesarios para la subsistencia física y cultural, la destrucción y contaminación del ambiente tradicional, entre otras consecuencias; por lo que en estos casos las decisiones de las comunidades pueden llegar a considerarse vinculantes, debido al grave nivel de afectación que les acarrea. (Sentencia T-769 de 2009).

En Colombia, la Consulta previa tiene un amplio desarrollo en medidas legislativas y administrativas. Para la Corte Constitucional (Sentencia C-366, 2011), las decisiones legislativas que puedan llegar afectar directamente a los pueblos indígenas deben ser consultadas previamente, si establezcan pautas que puedan llegar a tener sus consecuencias en las formas de vida de estos pueblos, además en congruen- 
cia con las obligaciones adquiridas en el Convenio 169 de la OIT. Asimismo, se debe consultar acerca de las medidas administrativas (Sentencia T-382, 2006), es decir aquellas que profieran las entidades que conforman la rama ejecutiva en su función administrativa; en este ámbito, la consulta se aplicará para aquellas situaciones en las cuales se ejecuten actos que puedan llegar afectar a los pueblos indígenas, por ejemplo la expedición de una licencia, concesiones o la contratación de una obra ${ }^{19}$.

A manera de ilustración, el caso emblemático en Colombia que permitiría evidenciar algunos vacíos de la consulta previa con relación a la confrontación de intereses económicos del Estado y los derechos de los pueblos indígenas, se encuentra en la dinámica de aplicación de la consulta en materia legislativa, específicamente en lo relacionado con la minería.

Actualmente, la minería representa una de las mayores preocupaciones para los indígenas ya que en el desarrollo de este sector, conlleva consecuencias irreparables que asumen directamente estos pueblos. Al mes de agosto de 2010, en el país había 8827 títulos oficialmente otorgados por el Instituto Nacional Geología y Minería a distintas empresas nacionales y extranjeras, así como a personas naturales. De tales títulos, según el propio Ministerio de Ambiente, al menos 571 (203 mil hectáreas) se refieren a zonas donde no se puede hacer minería de acuerdo con la legislación vigente, entre parques nacionales y regionales naturales, paramos, humedales y zonas de reserva forestal protectora (Ariza, 2013).

La política de Estado incentiva el crecimiento del sector minero, A través del reciente plan Visión 2019: Colombia como un país minero, el gobierno invitó y realizó diferentes acciones para favorecer las inversiones extranjeras y la creación de distritos mineros en buena parte del país (Ariza, 2013).
El Estado colombiano ha dado cumplimiento parcial a la adjudicación de tierras pertenecientes a pueblos indígenas, con lo que deja pendiente la legalización de la mayoría de las tierras, bajo la figura de Resguardo con título de propiedad colectiva. Se tiene presente que el título de propiedad colectiva connota de manera intrínseca el modo de tenencia ancestral de la tierras, vital para la garantía de la existencia física y cultural de los pueblos indígenas (Ariza, 2013, p. 16).

No obstante, en materia legislativa, la problemática es el Código Minero expedido a través de la Ley 685 de 2001, con una reforma en el año 2010, mediante la Ley 1382. Esta última fue declarada inexequible por la Corte Constitucional, a través de la Sentencia C-366 de 2011, debido a que desconoció el derecho de consulta de los pueblos indígenas y afrodescendientes de Colombia.

Por su parte, la Sentencia C-366 de 2011 afirma que:

Según los artículos 329 y 330 C.P., deberán estar sometidos al trámite de consulta previa los asuntos relacionados con la conformación, delimitación y relaciones con las demás entidades locales de las unidades territoriales de las comunidades indígenas; al igual que los aspectos propios del gobierno de los territorios donde habitan las comunidades indígenas; entre ellos la explotación de los recursos naturales en los mismos. Esto último según lo regulado por el parágrafo del artículo 330 C.P. el cual prevé que dicha explotación, cuando se realiza en los territorios indígenas, se hará sin desmedro de la integridad cultural, social y económica de las comunidades diferenciadas (Corte Constitucional de Colombia, sala plena, C-366, 2011).

Con relación a esta sentencia, la Corte concedió el término de dos años para que el Congreso de la República tomase las medidas legislativas dirigidas a la reforma del Código de Minas, una vez agotado un procedimiento 
de consulta previa a las comunidades indígenas y afrocolombianas, en los términos del artículo 330 de la Carta Política. De igual forma, se concede este mismo término para los efectos de la inconstitucionalidad de la Ley 1382 de 2010 y se excluye esta norma del ordenamiento jurídico colombiano.

Frente al contexto narrado, la problemática empeoró cuando, cumplido el periodo de dos años, el gobierno nacional expidió los decretos 933, 934, 935 y 943 de 2013, con lo que atendió a una ausencia total de consulta a los pueblos afectados, acompañó de una excesiva potestad reglamentaria y, en la práctica, se revivió el anterior Código Minero ${ }^{20}$.

Por su parte, entre otros aspectos que merecen tratarse, la Corte Constitucional ha dejado claro que el derecho de consulta no es absoluto y que las comunidades no tienen derecho a veto, lo que se justifica con el criterio del "interés general, en temas como el tratamiento de la minería y el sistema de regalías"21.

A finales del año 2015, en las bases de datos de la Corte Constitucional se encuentran 61 decisiones proferidas de la relación entre indigenas y consulta previa. Se puede la jurisprudencia de la Corte en lo relativo a: i) afectación a los indígenas respecto de las medidas legislativas del Estado; ii) afectación a los indígenas respecto de las medidas administrativas del Estado; y iii) afectación a los indígenas respecto de los proyectos de inversión y desarrollo del Estado. Allí puede se pueden ver terceros involucrados, como las empresas (Ortiz, 2016).

Entre los fallos más relevantes se pueden distinguir:

La Sentencia T-129 de 2011. Esta señaló que la consulta que se adelantaba ante los planes de desarrollo e inversión debía tener como finalidad el consentimiento libre previo e informado. Además, que la consulta no era un trámite, que debía hacerse con articulación y se orde- naba la suspensión de las actividades mineras concedidas por las entidades del Estado, hasta que se agotase efectivamente la consulta previa $\mathrm{y}$ se consiguiese finalmente el consentimiento de las comunidades.

La Sentencia T-693 de 2011, donde el tribunal insistió en que la consulta debía adelantarse frente a las medidas administrativas que adoptase el Estado, siempre y cuando estas incidieran y afectaran directamente en las comunidades. Es decir, cuando se visualizaran los impactos negativos, nacería la obligación de consulta.

La Sentencia C-331 de 2012, que aludió a la calidad de propietario del Estado frente al subsuelo y determinó que el deber de consulta solo se establecía mediante el método casuístico. Además dictaminó que debía verificarse cuáles eran las medidas que debían adoptarse en cada caso concreto. Luego, la Sentencia T-858 de 2013 determinó que los proyectos de exploración y explotación de recursos naturales debían ser consultados con las comunidades indígenas de conformidad con el art. 330 C.N.

La Sentencia T-384a de 2014 determinó que el proyecto debía socializarse ante la consulta; debían tomarse las medidas efectivas y tenerse en cuenta las medidas de afectación directa. Seguido, la Sentencia T-849 de 2014, dictaminó que la obligación de consulta garantizaba el derecho a la integridad cultural, igualdad y propiedad de los indígenas.

La Sentencia T-359 de 2015 estableció que la supervivencia de las comunidades indígenas y la preservación del medio ambiente debían garantizarse con la participación. De tal manera, este fallo señaló que las comunidades debían participar en el diseño y ejecución de actividades que pudiesen afectar su entorno. Asimismo, evitando la posible eliminación de los recursos, amparó los derechos de los indígenas y el derecho al medio ambiente. 
No obstante, a pesar del fructífero desarrollo jurisprudencial sobre la consulta previa por parte de la Corte Constitucional colombiana, en opinión de las autoras, aún resulta muy ambigüo determinar el alcance de la afectación directa de las comunidades indígenas. Todavía no existe un parámetro que permita medir dicha afectación y tal ambigüedad podría dar espacio a futuras vulneraciones de derechos en casos concretos.

\section{Consideraciones del derecho a la consulta previa: Colombia y Ecuador}

En Colombia y Ecuador se puede reconocer una inserción constitucional, legislativa y jurisprudencial de los lineamientos del Convenio 169 de la OIT sobre la consulta previa; sin embargo, vale la pena distinguir si dicha recepción se trató de una copia o tergiversación de la verdadera naturaleza de esta figura.

Una primera reflexión indica que, si en los casos planteados, el punto de partida hubiera sido la consulta con acompañamiento del consentimiento libre, previo e informado, esta figura del Derecho internacional hubiera cumplido su finalidad primaria de salvaguardar los derechos sustantivos de los pueblos indígenas. Derechos que llegaron a ser afectados por las medidas que tomó cada Estado en cuestión, a partir de sus decisiones legislativas o administrativas.

También es cierto que, en cada uno de estos casos seleccionados, la aplicación de la consulta previa está condicionada al "interés general", argumento que es utilizado por el máximo órgano jurisdiccional para mantener en firme una decisión de índole económica (Ley de Minería en Ecuador y Colombia) del gobierno de dichos Estados, frente a los intereses de protección de los pueblos indígenas. Valdría cuestionarse si en realidad los argumentos utilizados en el ordenamiento jurídico interno de cada Estado podrían llegar a traer como consecuencia una posible violación de las obligaciones adquiridas en el Convenio No. 169 de la OIT.
Ahora bien, sobre la problemática que conlleva el enfrentamiento de los intereses económicos del Estado, la consecuente explotación de recursos naturales y el derecho de participación de las poblaciones indígenas, en cuanto al proceso, deber y derecho de consulta, se evidencia que el Estado tiende a delegar sus obligaciones internacionales y nacionales a particulares.

En el caso colombiano, la realidad se despeja en proyectos donde intervienen agentes privados como las trasnacionales. De esta manera, se percibe una desatención por parte del Estado. Por ejemplo, el particular es quien termina realizando la consulta. Los agentes privados no lo hacen como producto de una concienciación en el respeto a los derechos de los grupos indígenas, sino de que "la consulta es un elemento derivado de su relación contractual" (Castillo, 2012, p. 7). Además, se percibe una ambigüedad mayor por parte del juez constitucional al tratar el grado de afectación de las decisiones sobre los pueblos indígenas, frente a la necesidad de ser consultados y dar cumplimiento a la finalidad de la figura jurídica.

Las realidades seleccionadas y estudiadas en este trabajo permiten recalcar el debate sobre las características que describen el desarrollo de los derechos de los pueblos indígenas a partir del diagnóstico de cada caso nacional. Los postulados de (2011), tratan sobre la generación de normativas en América Latina. Se aduce que los derechos de estas poblaciones tienen desarrollos distintos dependiendo el caso nacional. Es decir, si se analiza la situación de un Estado como Bolivia, se evidencia la incorporación decidida de la legislación pro indígena desde aspectos como el territorio y la importancia de los conocimientos tradicionales. En contraste, en Colombia el Estado ha reconocido la legislación indígena, pero afronta dificultades en hallar espacios de aplicación o acuerdos sobre sus alcances (Damonte, 2011). 


\section{Conclusiones}

Es importante resaltar que el proceso legislativo de los Estados seleccionados para este estudio refleja el crecimiento del ánimo de progreso económico por parte de sus gobiernos, lo que es necesario para el avance social de toda la población. Sin embargo, desde el punto de vista jurídico, pareciera que en el interior de su ordenamiento, los Estados presentan una incongruencia con los compromisos adquiridos en el plano internacional, al menos en lo relativo a su deber de consulta ante los pueblos originarios. Para estos nuevos retos resulta pertinente incentivar a la comunidad académica e investigativa a la revisión de las implicaciones de las obligaciones internacionales que asumen los Estados frente al deber de consulta de los pueblos indígenas y sus dinámicas internas en los ordenamientos jurídicos constitucionales de la realidad latinoamericana, ya que es precisamente allí donde se evidencian tensiones reales como la agenda legislativa de derechos sociales vs obligaciones internacionales de derechos humanos; o compromisos económicos internacionales vs progreso de derechos económicos, sociales y culturales; ambos asuntos que han sido ya revisados por estudios como los suscitados al interior del proyecto Ius Constitucionale Comune.

Ahora bien, pese a que los Estados adquieren las obligaciones del Convenio No. 169 de la OIT respecto a la consulta previa, en la actualidad no se ha encontrado la armonía entre los preceptos del orden jurídico internacional y la realidad jurídica interna de los pueblos indígenas. El desarrollo de los derechos de estos grupos étnicos en Latinoamérica se continuará trabajando en el plano internacional; sin embargo, bien vale la pena advertir que lo ideal sería una atención primaria y eficaz en el ámbito interno de cada Estado, que brindase todas las garantías y derechos para el desarrollo social, cultural y político de sus comunidades.

Se infiere que la participación política es una de las principales condiciones que necesi- tan los pueblos indígenas para el desarrollo de sus derechos y, en especial, para el ejercicio del derecho a la consulta previa. En los países caso de este estudio, la lucha por el derecho a la participación y consulta de estos pueblos se ejerce a través de una organización nacional. En Ecuador se encuentra la CONAIE y en Colombia la ONIC. Esta condición evidencia que, paralelo al desarrollo del deber de consulta de los Estados y su aplicación, hay un grupo de representantes de los pueblos indígenas debidamente organizados que fungiría como el principal defensor de sus propios intereses.

\section{Referencias}

Anaya, J. (2005). Los pueblos indigenas en el derecho internacional. Madrid, España: Trotta.

Antkowiak, T. (2014). Rights, Resources, and Rhetoric: Indigenous Peoples and the Inter-American Court. University of Pennsylvania Journal of International Law, 35(1), 113-187.

Ariza, R. (2013). Pueblos indígenas de Colombia ante el Sistema Interamericano de Derechos Humanos. Bogotá, Colombia: Universidad del Rosario. Recuperado de http://www.kas.de/wf/doc/kas_364171522-4-30.pdf?131216162331

Bogdandy, A., Salazar, P., Morales, M., y Ebert, F. (2018). El constitucionalismo transformador en América Latina y el derecho económico internacional. De la tensión al dialogo. México: Universidad Nacional Autónoma de México.

Carrión, P. (2012). Análisis de la consulta previa, libre e informada en el Ecuador. Quito, Ecuador: Konrad Adenauer.

Castillo, Y. (2012). El rol de la empresa transnacional extractiva de petróleo en la consulta previa con las comunidades indígenas: La experiencia en Colombia. Revista de Derecho, 37, 1-35. 
Damonte, G. (2011). Construyendo territorios: narrativas territoriales aymaras contemporáneas. Lima, Perú: Grade y Clacso.

DPLF y OXFAM. (2011). El derecho a la consulta previa, libre e informada de los pueblos indígenas. La situación de Bolivia, Colombia, Ecuador y Perú. Lima. Recuperado de http://www.dplf.org/sites/ default/files/1301596126.pdf

Figuera, S. (2015). Jurisdicción especial indigena en Latinoamérica. Una referencia especifica al Sistema Jurídico colombiano. Barranquilla, Colombia: Universidad del Norte y Grupo Editorial Ibáñez.

García, F. (2014). Territorialidad y autonomía, proyectos minero-energéticos y consulta previa: el caso de los pueblos indígenas de la Amazonía ecuatoriana. Antropológica, Año XXXII, 32, 71-85.

Hillón, Y. (2014). La consulta previa en la solución de conflictos socio-ambientales. Revista de Derecho, 41, 83-111.

Laurent, V. (2005). Presencia indígena en el paisaje político-electoral colombiano: balance y perspectivas. Women in Physics, The 2nd IUPAP International Conference on Women in Physics, Río de Janeiro, American Institute of Physics.

Morris, M., Rodríguez, C., Orduz, N., y Buriticá, P. (2010). La consulta previa a pueblos indígenas: los estándares del derecho internacional. Bogotá, Colombia: Programa de Justicia Global y Derechos Humanos, Universidad de los Andes.

OEA. (2016). Derechos de los Pueblos Indígenas y Tribales sobre sus Tierras Ancestrales y Recursos Naturales. Recuperado de https://www.oas.org/es/cidh/indigenas/ docs/pdf/Tierras-Ancestrales.ESP.pdf

OEA. (1996). Documentos preparatorios del Proyecto de Declaración Americana sobre los Derechos de los Pueblos Indígenas. Recuperado de http://www.cidh.org
Ortiz, M. (2016). El derecho a la integridad cultural de los pueblos indígenas frente a la responsabilidad social empresarial de las multinacionales mineras, en el marco del TLC Colombia-Estados Unidos. [Tesis de maestría]. Universidad del Norte, Barranquilla, Colombia. Recuperada de http://manglar.uninorte.edu.co/jspui/ bitstream/10584/7571/1/ortiz.pdf

Patiño, L. (2014). Fundamentos y práctica internacional del derecho a la consulta previa, libre e informada a pueblos indígenas. Anuario Colombiano de Derecho Internacional, 7, 69-111. doi: 10.12804/ acdi7.2014.03

Rodríguez, C., y Orduz, N. (2012). La consulta previa: dilemas y soluciones. Lecciones del proceso de construcción del decreto de reparación y restitución de tierras para pueblos indígenas en Colombia. Bogotá, Colombia: Centro de Estudios de Derecho, Justicia y Sociedad, Dejusticia.

Rodríguez Van der Hammen, A. (2013). El derecho de las comunidades étnicas a la consulta previa y al consentimiento libre, previo e informado: desarrollo jurisprudencial y retos para su implementación. Bogotá, Colombia: Universidad de los Andes.

Rodríguez, G. (2017). De la consulta previa al consentimiento libre, previo e informado a pueblos indigenas en Colombia. Bogotá, Colombia: Grupo Editorial Ibáñez y GIZ. doi: 10.12804/tj9789587389081

Rodríguez-Piñero, L. (2007). Pueblos indigenas y Derecho internacional: una historia incómoda. Ciudadanía y derechos indígenas en América Latina: poblaciones, Estados y orden internacional. Madrid, España: Centro de Estudios Políticos y Constitucionales.

Saavedra, L. (2011). Ecuador ¿Consulta o Consentimiento Previo? En: Noticias aliadas, Lima, Edición especial Consulta 
Previa: Derecho fundamental de los pueblos indígenas.

Schilling-Vacaflor, A., y Flemmer, R. (2013). Why is Prior Consultation Not Yet an Effective Tool for Conflict Resolution? The Case of Peru. Hamburgo: German Institute of Global and Area Studies.

\section{Jurisprudencia}

Corte Interamericana de Derechos Humanos. (17 de junio de 2005). Caso Comunidad Indígena Yakye Axa Vs. Paraguay. Serie C; $\mathrm{N}^{\circ} 125$, (Fondo, Reparaciones y Costas). Recuperado de http://www. corteidh.or.cr/docs/casos/articulos/ seriec_125_esp.pdf

Corte Interamericana de Derechos Humanos. (31 de agosto de 2001). Caso de la Comunidad Mayagna (Sumo) Awas Tingni vs. Nicaragua. Serie C, N 79, (Fondo, Reparaciones y Costas). Recuperado de http:// www.corteidh.or.cr/docs/casos/articulos/ Seriec_79_esp.pdf

Corte Interamericana de Derechos Humanos. (15 de junio de 2005). Caso de la Comunidad Moiwana vs. Suriname. Serie C, $\mathrm{N}^{\circ}$ 124, (Excepciones Preliminares, Fondo, reparaciones y Costas). Recuperado de http://www.corteidh.or.cr/docs/casos/ articulos/seriec_124_esp.pdf

Corte Interamericana de Derechos Humanos. (27 de junio de 2012). Caso Pueblo Indígena Kichwa de Sarayaku vs. Ecuador. Serie C, No 245, (Fondos y Reparaciones). Recuperado de http://corteidh.or.cr/ docs/casos/articulos/seriec_245_esp.pdf

Corte Interamericana de Derechos Humanos. (8 de octubre de 2015). Caso de la Comunidad Garífuna de Punta Piedra y Sus Miembros vs. Honduras. Serie C, N 304, (Excepciones Preliminares, Fondo, reparaciones y Costas). Recuperado de http:// www.corteidh.or.cr/docs/casos/articulos/ seriec_304_esp.pdf
Corte Constitucional Colombia. (9 de abril de 1996). Sentencia C-139. [MP Carlos Gaviria Díaz]

Corte Constitucional Colombia. (22 de mayo de 2006). Sentencia T-382. [MP Clara Vargas]

Corte Constitucional de Colombia. (13 de febrero de 2013). Sentencia C-891. [MP Luis Guillermo Guerrero]

Corte Constitucional de Colombia. (11 de mayo de 2011). Sentencia C-366. [MP Luis Ernesto Vargas Silva]

Corte Constitucional de Ecuador. (18 de marzo de 2010). Sentencia No. 001-10-SINCC. [ $\mathrm{JCP}^{22}$ : Patricio Pazmiño Freire]

\section{Notas}

1 En este trabajo se entenderá por pueblos indígenas y otros grupos étnicos lo expuesto por el Convenio No. 169 de la OIT en su artículo 1o. inciso 3. Organización Internacional del Trabajo, "Convenio No. 169 sobre Pueblos Indígenas y Tribales en Países Independientes", traducción en español. Recuperado de: http://www.ilo.org/indigenous/Conventions/no169/lang--es/index. htm. Acceso: 15 de abril de 2015.

2 Sin embargo, cabe destacar que con anterioridad al Convenio No. 169 de la OIT se encuentra el Convenio No. 107 de 1957 y la Recomendación No. 104, ambos igualmente emanados de la OIT, los cuales figuran como los primeros estándares jurídicos en el plano internacional, en materia de reconocimiento de los derechos de los pueblos indígenas en países independientes. Para ampliar sobre los aportes del Convenio No. 107 de la OIT y sus aportes como modelo integracionista en el desarrollo de los pueblos indígenas, ver Rodríguez-Piñero, Luis, "Pueblos indígenas y Derecho internacional: una historia incómoda", Ciudadanía y derechos indígenas en América 
Latina: poblaciones, Estados y orden internacional, Madrid, Centro de Estudios Políticos y Constitucionales, 2007, pp. 83-105.

3 Sobre el deber de consulta que tienen de los Estados en relación con sus pueblos indígenas, cfr. Organización Internacional del Trabajo, "Convenio No. 169 sobre Pueblos Indígenas y tribales...", Organización Internacional del Trabajo, "Convenio No. 169 , artículo $6^{\circ}$.

4 En el sistema interamericano de derechos humanos, los derechos territoriales de los pueblos indígenas tienen su fundamento en el artículo XXIII de la Declaración Americana de los Derechos y Deberes del Hombre y en el artículo 21 de la Convención Americana sobre Derechos Humanos. Estos artículos no hacen remisión expresa a los derechos de los pueblos indígenas, sin embargo, la Comisión interamericana y la Corte Interamericana han hecho su labor de interpretación en sentido que protege los derechos que tienen estos pueblos y sus miembros sobre su territorio y sus recursos naturales. OEA, Derechos de los Pueblos Indígenas $y$ Tribales sobre sus Tierras Ancestrales $y$ Recursos Naturales, Recuperado de: http:// cidh.org/countryrep/Tierras Indigenas2009/ Cap.I-II.htm

5 La Declaración Americana sobre los derechos de los pueblos indígenas fue un proyecto entregado por la Comisión Interamericana de Derechos Humanos en 1997, la cual fue aprobada por la OEA mediante la Resolución AG/RES. 2888 (XLVI-O/16) en la segunda sesión plenaria, celebrada el 14 de junio de 2016. Ver Morris et al., 2009, p. 32.

6 Esta función consiste en que recibir denuncias de individuos sobre violaciones a sus derechos por parte del Estado. En tales casos, puede intentar llegar a una solución amistosa entre los Estados y la parte denunciante $\mathrm{o}$, en caso de fallar esta solución, puede remitir el caso a la Corte Interame- ricana si el Estado implicado ha reconocido la competencia de ella. Así también, la comisión tiene la posibilidad de conceder medidas cautelares por iniciativa propia o por petición de parte para evitar daños irreparables (Morris et al., 2009, pp. 32-33).

7 Cabe aclarar que en este caso, la Corte estimó que los Saramkas no son pueblos indígenas. Sin embargo, por compartir algunas características de los indígenas, como su identificación con su territorio tradicional y la administración (en parte) autónoma según normas, costumbres y tradiciones propias la Corte IDH dictaminó que su jurisprudencia para pueblos indígenas también sería aplicable a los Saramakas. Debido a que Surinam no ratificó el Convenio 169 de la OIT, los derechos de esta comunidad tribal se derivaron de los tratados internacionales de derechos humanos y de la Convención Americana sobre Derechos Humanos. Ver: Schilling-Vacaflor, Almut y Flemmer, Riccarda, Why is Prior Consultation Not Yet an Effective Tool for Conflict Resolution? The Case of Peru, Hamburgo, German Institute of Global and Area Studies, 2013, p.18.

8 A la fecha 22 ratificantes. Recuperado de http://www.ilo.org

9 Recuperado de http://www.ilo.org

10 En el artículo 84 numeral 5 expresó: "ser consultados sobre planes y programas de prospección y explotación de recursos no renovables que se hallen en sus tierras y que puedan afectarlos ambiental o culturalmente; participar en los beneficios que esos proyectos reporten, en cuanto sea posible y recibir indemnizaciones por los perjuicios socio-ambientales que les causen".

11 En el artículo 57 numeral 7 expresó “La consulta previa, libre e informada, dentro de un plazo razonable, sobre planes y programas de prospección, explotación y comercialización de recursos no renovables que se encuentren en sus tierras y que puedan 
afectarles ambiental o culturalmente; participar en los beneficios que esos proyectos reporten y recibir indemnizaciones por los perjuicios sociales, culturales y ambientales que les causen. La consulta que deban realizar las autoridades competentes será obligatoria y oportuna. Si no se obtuviese el consentimiento de la comunidad consultada, se procederá conforme a la Constitución y la Ley."

12 "El término Pachamama se encuentra formado por los vocablos Pacha que en quechua significa universo, mundo, tiempo, lugar, y Mama, traducido como madre. Hay acuerdo entre varios autores en considerar a Pachamama como una deidad andina que en su aspecto simbólico se relaciona con la tierra, la fertilidad, la madre, lo femenino". Diccionario de Mitos y Leyendas, recuperado de http://www.cuco.com.ar/pacha mama.htm

13 Según los artículos 24 y 26 del Decreto 3401 de 2002 se contempla la posibilidad de hacer convocatoria de consulta a través de los representantes de las comunidades indígenas, sin involucrar a la totalidad de sus miembros. Ver Saavedra, 2011, p. 6.

14 El decreto 1040 establece que la opción de una comunidad frente a un proyecto solo podrá ser considerada siempre y cuando sea técnica y económica viable, es decir, siempre y cuando compense económicamente las ganancias que una empresa dejará de recibir al no explotar los recursos de un territorio. Ver Saavedra, 2011, p.6.

15 La Corte Constitucional del Ecuador indicó que "la consulta pre legislativa es un derecho colectivo, que está dirigido única y exclusivamente a las comunas, comunidades, pueblos y nacionalidades indígenas, afroecuatorianos y montubios que puedan verse afectados en sus derechos colectivos, de manera práctica y real por efectos de aplicación de una ley". Corte Constitucional de Ecuador, Sentencia
No. 001-10-SINCC. Juez Constitucional Ponente: Patricio Pazmiño Freire (18 de marzo, 2010). Ecuador, 2010.

16 DANE, "Censo general". Recuperado de http://www.dane.gov.co/index.php/esp/ poblacion-y-registros-vitales/censos/censo-2005

17 Ver artículos 171 y 286 de la Constitución Política de Colombia.

18 Artículos 246, 321, 329 y 330 de la Constitución Política de Colombia.

19 Sobre un amplio estudio la consulta previa en medidas legislativas y administrativas. Véase Rodríguez, G., 2014, pp. 65-107.

20 De acuerdo con Juan José Parada Holguín, jefe de la Oficina Asesora Jurídica del Ministerio de Minas y Energía: "esos decretos están reglamentando la Ley 685 del 2001, que ya fue consultada previamente con las comunidades étnicas, y teniendo en cuenta que son actos reglamentarios de una norma ya consultada, por concepto de la Corte Constitucional, se entiende que los actos administrativos reglamentarios no requieren de la consulta previa". Recuperado de http://www.ambitojuridico.com

21 Ver: Corte Constitucional de Colombia, C-891, 2002, sobre el análisis de constitucionalidad de la Ley 685 de 2001; Corte Constitucional Colombia, C-068, 2013, sobre la Ley 1530 de 2012 sistema de regalías.

22 Se entiende como Juez Constitucional Ponente. 
